\title{
Runt sturgeon - the case study of abnormal growth in Acipenseridae juveniles
}

\author{
Dobrochna Adamek-Urbańska, Katarzyna Jabłońska, Małgorzata Rzepkowska, \\ Magdalena Fajkowska, Jerzy Śliwiński, Teresa Ostaszewska
}

Received - 14 January 2020/Accepted - 26 April 2020. Published online: 30 June 2020; @Inland Fisheries Institute in Olsztyn, Poland Citation: Adamek-Urbańska D., Jabłońska K., Rzepkowska M., Fajkowska M., Śliwiński J., Ostaszewska T. 2020 - Runt sturgeon - the case study of abnormal growth in Acipenseridae juveniles - Fish. Aquat. Life 28: 73-76

\begin{abstract}
The occurrence of runt sturgeon in stocks reared in recirculating aquatic systems is highly undesirable for both production and fish welfare. The aim of this study was to describe the anatomopathological and histopathological changes accompanying the phenomenon of runt sturgeon reared in fully controlled recirculating aquatic conditions. These individuals were characterized by emaciation and cachexia. Histopathological analysis indicated severe chronic non-purulent inflammation of the gastrointestinal tract and exfoliation of enterocytes into the lumen, accompanied by chronic, non-purulent pancreatitis. All pathological findings were probably the result of malnutrition, which further escalated into the disorders observed.
\end{abstract}

Keywords: abnormal growth, cachexia, histopathology, sturgeon

\section{Introduction}

Diversified growth in aquaculture is an undesirable phenomenon that generates additional costs resulting from feed loss and additional labor costs related

D. Adamek-Urbańska [ $\left.\Xi^{\circ}\right]$, K. Jabłońska, M. Rzepkowska,

M. Fajkowska, J. Śliwiński, T. Ostaszewska

Department of Ichthyology and Biotechnology in Aquaculture,

Institute of Animal Science, Warsaw University of Life Sciences.

Ciszewskiego 8, 02-786, Warsaw, Poland

Tel:+48225936640; e-mail: dobrochna_adamek@sggw.pl to segregating smaller animals. It is assumed that some growth abnormalities are related to hierarchy in sturgeon cohorts leading to subordinate individuals having limited access to food (Domeneghini et al. 2002, Di Giancamillo et. al. 2012) and other currently unknown etiological factors. In natural conditions, where starvation is temporary, compensatory growth is observed after periods of malnutrition (Liu et al. 2011). However, prolonged starvation can cause irreversible nutritional deficiencies resulting in the escalation of histopathological changes, which are observed to spread further in numerous tissues. Regardless of whether the starvation period is short or long, inflammation can occur in the gastrointestinal tract and associated glands, such as the liver and pancreas (Kongtrop et al. 2014). This can lead to fish being more susceptible to infection, which can be caused not only by pathogenic, but also opportunistic agents. Nevertheless, sturgeon growth disorders are still poorly understood. Therefore, the aim of this study was to describe the anatomopathological and histopathological changes accompanying the phenomenon of runt sturgeon.

\section{Material and methods}


Seven Russian sturgeon, Acipenser gueldenstaedtii Brandt \& Ratzeburg (four runt, three control) individuals, reared in fully controlled indoor recirculating aquatic conditions and at 130 days post hatching (dph), were selected from a cohort because they exhibited apathy and significantly decreased body size. In the runt and control individuals, the average body weight was $31.64 \pm 6.56$ and $48.62 \pm 6.72 \mathrm{~g}$ and total body length was $22.53 \pm 0.71$ and $26.80 \pm 1.05$ $\mathrm{cm}$, respectively. On the day of selection, and before sampling, the fish were fed a commercial diet (Skretting). The feeding regime and rearing parameters were in accordance with Rzepkowska et al. (2014). The sampled fish were anesthetized (MS-222, Sigma Aldrich), weighed, and measured. The gastrointestinal tract (stomach and all intestinal parts) and pancreas were collected for histological analyses. The tissues were fixed in Bouin's solution and subsequently subjected to standard histological procedures. Sections (5 $\mu \mathrm{m}$ thick) were stained with hematoxylin and eosin (HE).

\section{Results}

All the runt individuals were characterized by emaciation and cachexia. Local petechiae were observed in the skutes and the skin. The pancreases were visibly altered, spotted, and lacking the structure and consistency of healthy organs. The gastrointestinal tract was empty. The histological picture indicated chronic non-purulent pancreatitis, and the pancreas was completely occupied by immune cells (mainly lymphocytes, single histiocytes, and granulocytes), perivascular fibrosis was evident, and there were no normal acinar cells (Fig. 1A), none of these were observed in the normal pancreases of the healthy individuals (Fig. 1B). All of the runt fish examined exhibited numerous infiltrations of immune cells (lymphocytes, monocytes, granulocytes) and variable insensitivity of mucosa desquamation in all parts of the gastrointestinal tract (Fig. 1C-F), which were indicated by the typical histological picture of chronic, non-purulent gastroenteritis. The intestinal folds in the runt fish were characterized by local glandular epithelium metaplasia with atrophy in the supranuclear apical area of the enterocytes (Fig. $1 \mathrm{E}-\mathrm{F})$. Degenerating myocytes with cytoplasmic vacuolization and pyknotic nuclei were observed pervasively in the smooth muscular layer of all the gastrointestinal tract sections (Fig. 1G-H).

\section{Discussion}

Aquaculture, similarly to livestock breeding, is focused on the intensive production of animal products. In fish production, cohorts are often characterized by uneven growth. Individuals with lower body weights compared to the stock average are usually subjected to selection. Sometimes large growth rate disproportions among specimens of the same sturgeon stock are observed despite providing optimal environmental conditions and balanced diets for given species. Previous research conducted on sturgeon has shown that behavior is also an important factor influencing growth (Georgiadis et al. 2000).

During this study, the desquamation of the mucous membrane, which is the largest absorbent surface in the gastrointestinal tract, was detected in the runt sturgeon examined. This type of lesion is characteristic of chronic gastroenteritis, which can be caused by multiple etiological factors, for example malnutrition (Georgiadis et al. 2000, Di Giancamillo et al. 2012), parasites (Esch and Huffines 1973), bacteria (Song et al. 2014), or viruses (Muroga 2001). Similarly, histopathological examinations of white sturgeon, Acipenser transmontanus Richardson with abnormal growth rates showed the presence of catarrhal to desquamating esophagogastroenteritis, increased intestinal thickness, reductions in the height of intestinal folds, and numerous other histopathological changes (Di Giancamillo et al. 2012). Because of the variety of factors that could be responsible for the changes observed in the current study, it is hard to state unambiguously what their main cause was; however, the most probable cause 


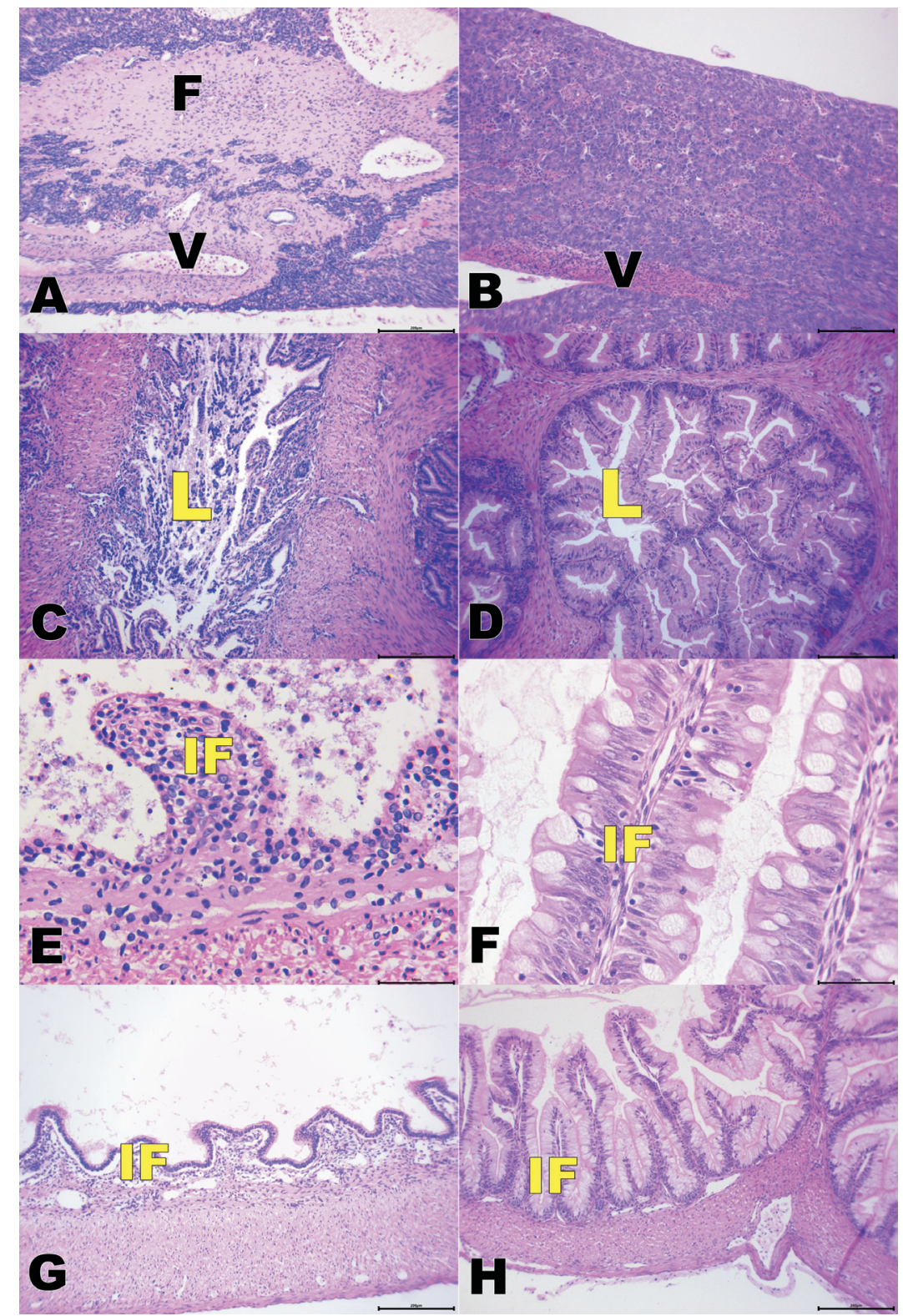

Fig. 1. Histopathological changes in the digestive tract of runt sturgeon (A, C, E, G) compared to control individuals (B, D, F, H): A - pancreas - perivascular fibrosis; B - control pancreas; $\mathrm{C}$ - pyloric ceca with a mass of exfoliated cells from the mucous membrane in the lumen; D - control pyloric ceca; E - infiltration of inflammatory cells in the submucosa and desquamated mucosa of the intestinal fold; F normal intestinal fold with enterocytes and mucosa; $\mathrm{G}$ - spiral valve with degenerated intestinal fold mucosa; $\mathrm{H}$ - normal spiral valve with intestinal folds; F - fibrosis, V - blood vessel, L - lumen, IF - intestinal fold. HE staining. Scale bars - $200 \mu \mathrm{m}(\mathrm{A}-\mathrm{D}, \mathrm{G}-\mathrm{H}), 50 \mu \mathrm{m}(\mathrm{E}, \mathrm{F})$.

was long term malnutrition. Pancreatitis is usually accompanied by multi-organ inflammation involving the entire abdominal cavity (Hoole et al. 2008), which was also observed in the fish analysed. A number of etiological factors can cause the development of pancreatitis (Kongtorp et al. 2004), one of which is a salmonid viral disease of the pancreas. This disease is also found in many fish species and yields similar macro and microscopic pictures to the ones observed in this study. However, etiological factors of diseases are poorly recognized in sturgeon. It is noteworthy that the sturgeon analyzed were reared in indoor recirculating aquatic system tanks that limit the possibility of fish infection.

To conclude, the multi-organ analyses performed in this study revealed that runt sturgeon not 
only exhibit uneven growth but also a number of histopathological changes. The extensive histopathological changes observed most probably resulted from malnutrition, which subsequently caused organ dysfunction and triggered immune responses. Nevertheless, further more detailed analyses are required to identify all the causes of the runt sturgeon phenomenon.

Acknowledgments. This work was supported financially by the Faculty of Animal Sciences, Warsaw University of Life Sciences.

Conflict of Interest. The authors declare that they have no conflicts of interest.

Ethical approval. All applicable international, national, and/or institutional guidelines for the care and use of animals were followed by the authors.

Author contributions.

ORCID ID

Dobrochna Adamek-Urbańska.

(iD https://orcid.org/0000-0002-3840-935X

\section{References}

Di Giancamillo A., Martino P.A., Arrighi S., Domeneghini C. 2012 - Gut peculiarities of feed deprived white sturgeons (Acipenser transmontanus, Richardson 1836) - Open J. Vet. Med. 2: 52-59.

Domeneghini C., Radaelli G., Bosi G., Arrighi S., Di Giancamillo A., Pazzaglia M., Mascarello F. 2002 -
Morphological and histochemical differences in the structure of the alimentary canal in feeding and runt (feed deprived) white sturgeons (Acipenser transmontanus) - J. Appl. Ichthyol. 18: 341-346.

Esch G.W., Huffines W.J. 1973 - Histopathology associated with endoparasitic helminths in bass - J. Parasitol. 59: 306-313.

Georgiadis M.P., Hedrick R.P., Johnson W.O., Yun S., Gardner I.A. 2000 - Risk factors for outbreaks of disease attributable to white sturgeon iridovirus and white sturgeon herpesvirus-2 at a commercial sturgeon farm - Am. J. Vet. Res. 61: 1232-1240.

Hoole D., Bucke D., Burgess P., Wellby I. 2008 - Diseases of carp and other cyprinid fishes - Blackwell Science Publishing, Oxford, UK.

Kongtorp R.T., Taksdal T., Lyngřy A. 2004 - Pathology of heart and skeletal muscle inflammation (HSMI) in farmed Atlantic salmon Salmo salar - Dis. Aquat. Organ. 59: 217-224.

Liu W., Wei Q.W., Wen H., Jiang M., Wu F., Shi Y. 2011 Compensatory growth in juvenile Chinese sturgeon (Acipenser sinensis): effects of starvation and subsequent feeding on growth and body composition - J. Appl. Ichthyol. 27: 749-754.

Muroga K. 2001 - Viral and bacterial diseases of marine fish and shellfish in Japanese hatcheries - Aquaculture 202: 23-44.

Rzepkowska M., Ostaszewska T., Gibala M., Roszko M.L. 2014 - Intersex gonad differentiation in cultured Russian (Acipenser gueldenstaedtii) and Siberian (Acipenser baerii) sturgeon - Biol. Reprod. 90: 1-10.

Song S.K., Beck B.R., Kim D., Park J., Kim J., Kim H.D., Ringø E. 2014 - Prebiotics as immunostimulants in aquaculture: a review - Fish Shellfish Immun. 40: 40-48. 